\title{
A Combined Effect of Electron Beam and Stress on Plastic Flow of Amorphous Silica Microparticles.
}

\author{
Sanjit Bhowmick, Douglas Stauffer, Ryan Major, Oden Warren and S. A. Syed Asif
}

Hysitron, Inc., Minneapolis, Minnesota 55344, USA

Radiation induced plastic flow in amorphous silica glass is an important subject in glass science and technology and have been studied for decades by many researchers using high energy ions and particles. However, the deformation behavior of such material irradiated by low energy electrons is not well understood. In comparison to heavier particles and ions, electrons have much higher penetration depths and therefore can generate uniform damage and structural changes throughout the sample. In this study, we investigate plastic flow of silica particles under a combined effect of compressive stress and electron beam inside a scanning electron microscopy. To prepare the particle samples for compression experiments, silica microparticles of diameter $\sim 1 \mu \mathrm{m}$ were mixed in water, ultrasonicated for 10 minutes, and dispersed on silicon substrates. In situ compression experiments were conducted using a PI 85 SEM PicoIndenter (Hysitron, Inc., Minneapolis, MN) with $5 \mu \mathrm{m}$ flat punch diamond probe. TriboScan software was used to record and analyze load-displacement data. The load-displacement plots and real-time video of deformation were synchronized and captured during the experiment, which aided post-experimental analysis. Quasistatic compression experiments were conducted at $P_{\max }=0.05 \mathrm{mN}, 1$ $\mathrm{mN}$ and $4 \mathrm{mN}$ under different beam intensities.

Figures 1a-f show deformation behavior of the particles before and after the experiments with beam off and beam on conditions. Considerable variation in the plastic strain has been observed with peak loads and beam condition. In these tests, plastic strain was calculated as $\delta / D$, where $D$ is the diameter of the particle and $\delta$ is the amount of compression along the indentation axis. At $1 \mathrm{mN}$ load and beam off condition, a particle showed negligible strain $(<0.05 \%)$, fig. 1a. However, similar diameter particles deformed plastically to $47 \%$ and $67 \%$ strain when the beam was kept on and probe currents of $30 \mathrm{pA}$ and $480 \mathrm{pA}$ were applied (fig. $1 \mathrm{~b}$ and c). In another set of experiments at $4 \mathrm{mN}$ peak load and beam off condition, a particle deformed plastically to $36 \%$ strain and fractured creating a wedge-shaped missing segment (fig. 1d). When an electron beam with probe current of $30 \mathrm{pA}$ was applied, a similar particle deformed plastically to $58 \%$ strain with a surface crack (fig. 1e). However, no crack was observed when a high probe current of $480 \mathrm{pA}$ was applied during a test (fig. 1f). It is important to note that all the particles used in this study were exposed to electron beam before starting a test. So, it can be assumed that irradiation induced damage or defects in all the particles before loading were similar. This leads to a conclusion that both the applied load and beam energy are playing significant role in enhancing the structural changes which causes large plastic flow. Electron beam also shows an instantaneous effect on plastic flow as illustrated in figure $1 \mathrm{~g}$. The total energy consumed by a particle during a test can be considered as $U_{t}=U_{e}$ (elastic deformation energy) $+U_{p}=$ plastic deformation energy $+U_{r}=$ beam energy. The variation of strain as a function of load and beam intensity is shown in fig. $1 \mathrm{~h}$. Densification, anisotropic deformation, and radiation induced plastic flow will be discussed as possible deformation mechanism in the presentation. An important implication of this study is that electron irradiation under applied stress can induce significant instability and reduction in strength in silica resulting in lower lifetime in many devices where silica is an integral component. 


\section{References:}

[1] P. M. Ajayan and S. Iijima, Phil. Mag. Lett., 65 (1992), p. 43.

[2] D. Stauffer and S. Bhowmick, R. Major, O. Warren and S. Asif, Microscopy and Microanalysis, 20 (2014), p. 1544.

[3] K. Zheng, Nature Communication, 1 (2010), p 1.

[4] E. Snoeks, A. Polman and C. A. Volkert, APL, 65 (1994), p 112646.
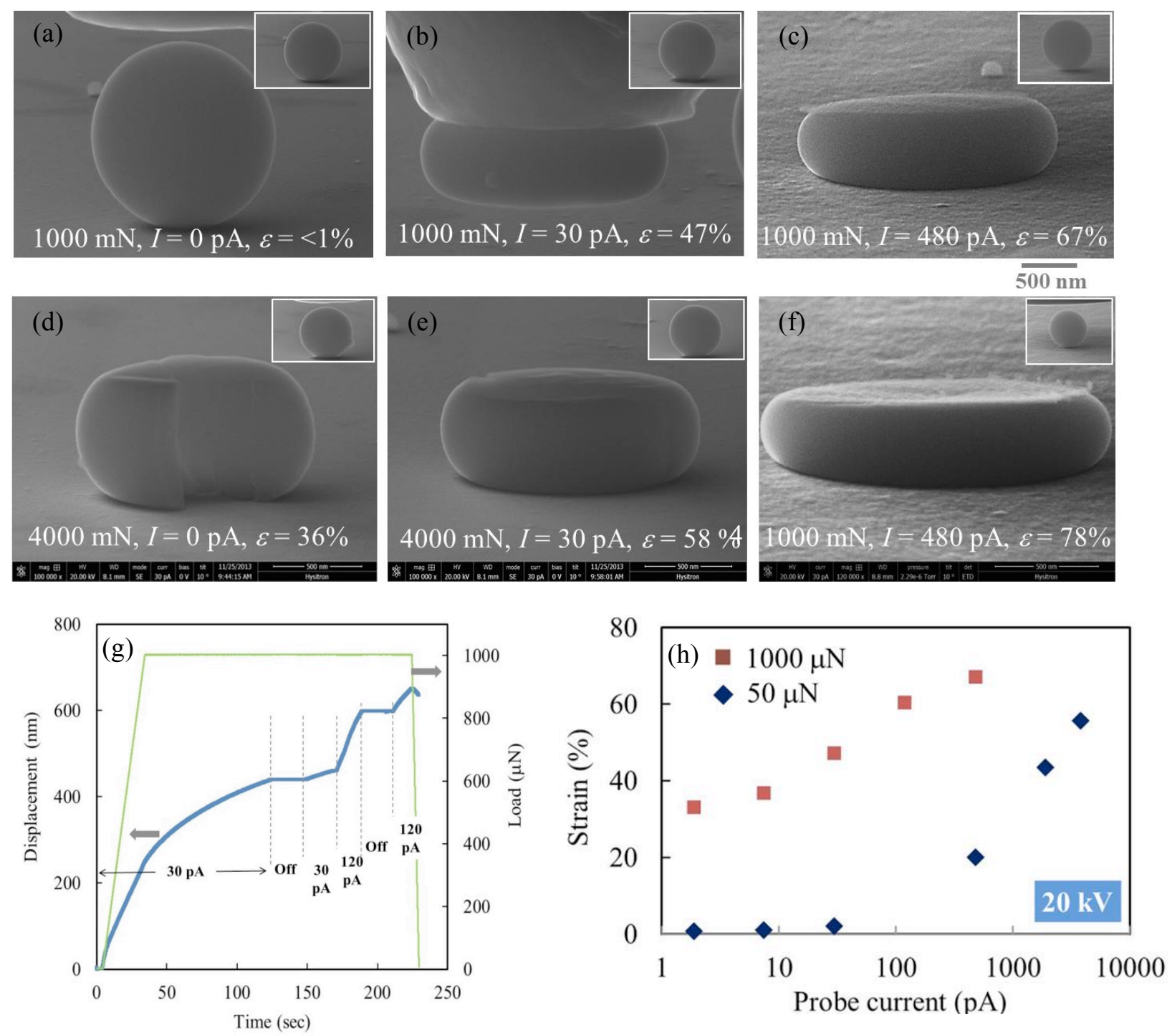

Figure 1: a-f: Images of silica particles after compression with beam off (a and d) and beam on (b, c, e, f) conditions. Fig. g shows instantaneous effect of beam off, beam on, and variation of probe current. Fig. h shows plastic strain as a function of load and beam intensity. 